\title{
Feasibility of an Online Mindfulness-Based Intervention for Women with Sexual Interest/Arousal Disorder
}

\author{
Lori A. Brotto' $\cdot$ Kyle R. Stephenson ${ }^{2}$ Natasha Zippan ${ }^{3}$ \\ Accepted: 18 December 2021 / Published online: 4 January 2022 \\ (c) The Author(s), under exclusive licence to Springer Science+Business Media, LLC, part of Springer Nature 2022
}

\begin{abstract}
Objectives Difficulties with sexual desire impact up to a third of women and most do not seek or receive appropriate care for these complaints, in part due to stigma, embarrassment, and limited availability of treatment. In-person mindfulnessbased interventions have lasting benefits to sexual desire and sex-related distress in controlled clinical trials but are difficult to access. Online cognitive-behavioral interventions for sexual concerns have shown promising findings, but online mindfulness interventions have received little testing. The current study assessed the feasibility of an online program (called eSense-Mindfulness) adapted from an effective face-to-face intervention for women with Sexual Interest/Arousal Disorder. Methods Thirty cisgender women $(M$ age $=35.3)$ with Sexual Interest/Arousal Disorder consented and completed at least one of the eight modules of $e$ Sense-Mindfulness while providing weekly feedback regarding their experience. Feasibility was assessed via attrition rates and participant self-report regarding challenges of using the program. Limited efficacy testing was based on effect sizes for changes in sexual response and sex-related distress.

Results Participants ( $n=25$ who completed all testing) reported high levels of usability and ease of understanding content and reported the mindfulness exercises as well as the information on partner communication to be the most relevant. Limited efficacy testing showed large effect sizes for reductions in sex-related distress, and improvements in sexual desire, arousal, and satisfaction whereas there were smaller effect sizes for improvements in lubrication, orgasm, and vaginal pain.

Conclusions The findings suggest that efficacious face-to-face mindfulness interventions for sexual dysfunction in women show excellent evidence of feasibility when delivered online without personalized guidance.
\end{abstract}

Keywords Mindfulness $\cdot$ Sexual interest/arousal disorder $\cdot$ Sexuality $\cdot$ Internet interventions $\cdot$ Online therapy $\cdot$ Telehealth

Low sexual desire is extremely common in women, impacting up to a third of women in large, representative, and cross-sectional studies (Mitchell et al., 2013). When combined with clinically significant distress, the disorder is officially classified in the Diagnostic and Statistical Manual of Mental Disorders (DSM-5; American Psychiatric Association, 2013) as female sexual interest/arousal disorder (SIAD). SIAD is diagnosed when any three of six polythetic

Lori A. Brotto

Lori.Brotto@vch.ca

1 Department of Obstetrics and Gynaecology, University of British Columbia, 2775 Laurel Street, Vancouver, BC V5Z 1M9, Canada

2 School of Psychology, Xavier University, Cincinnati, OH 45207, USA

3 Department of Psychology, University of British Columbia, Vancouver, BC V6T 1Z4, Canada criteria are present for at least 6 months (APA, 2013) and may include lack of interest (or no interest) in sexual activity; reduced or absent erotic thoughts or fantasies; reduced level of initiating sex and/or responding to a partner's sexual advances; reduced pleasure during sexual activity; lack of responsive sexual desire (desire that emerges with or after sexual arousal); and reduced genital and nongenital sexual sensations (i.e., arousal). A population-based study that evaluated SIAD criteria combined with the 6-month duration plus clinically significant distress indicated a population prevalence of $0.6 \%$ (Mitchell et al., 2016), although ongoing low sexual desire with subclinical subjective distress is much more common (Mitchell et al., 2013).

Dewitte's (2016) emotion-motivational model of sexual response is useful for describing potential causal and maintaining factors of low sexual desire and also suggests possible underlying mechanisms of action for related treatments. The model states that sexual response is triggered by 
a stimulus that pre-attentively captures attention and is automatically appraised as sexually meaningful and rewarding. These automatic appraisals lead to increased cognitive focus as well as a genital sexual response (e.g., vaginal lubrication). Both automatic and conscious appraisals interact with attentional processes and can lead to increased and deliberate attention to a sexual stimulus. If the outcome is a positive evaluation, then a positive subjective sexual response emerges, and could further increase physical arousal. These sexual responses may then increase the motivation to engage in sexual activities (Dewitte, 2016). In accounting for low desire, Dewitte's model predicts that low desire results when a person fails to notice a sexual stimulus, their attention is diverted by non-sexual distractions, or when a sexual stimulus is not appraised as rewarding. Thus, attention and distraction should be primary targets in an effective treatment for SIAD.

One key source of distraction from sexual stimuli can be negative beliefs and thoughts about sex. Compared to women without sexual difficulties, women with low sexual desire hold stronger negative sex-related beliefs (e.g., that aging confers negative effects on body image and sexuality), and this makes women more vulnerable to activation of negative self-schemas, resulting in more frequent negative automatic thoughts in sexual situations (Nobre \& Pinto-Gouveia, 2006). Once elicited, these negative automatic thoughts can prevent women from focusing on relevant sexual stimuli, which further interferes with sexual response. As a result of these elicited negative thoughts and emotions, women may avoid sexual situations and even contexts that can lead to possible sexual encounters (such as non-sexual intimate activities; Barlow, 1986).

Chivers and Brotto (2017) postulated that mindfulnessbased treatments, with their focus on guided attention, acceptance, and self-compassion, might be especially suitable for addressing these common negative thoughts and feelings characteristic of women with SIAD, and for addressing the importance of attentional factors as outlined in the emotion-motivational model (Dewitte, 2016). Mindfulness-based approaches have emerged as the "third wave" of cognitive-behavioral therapies (CBT) and aim to cultivate active awareness of one's present experience in an accepting, nonjudgmental, and compassionate manner. A number of small studies have evaluated mindfulness-based approaches for sexual dysfunction in women (Brotto \& Heiman, 2007; Stephenson \& Kerth, 2017) with three-session (Brotto, et al., 2008a, b) and four-session (Brotto \& Basson, 2014) group mindfulness programs showing efficacy for improving sexual desire. Eight-session versions that more closely align with Mindfulness-Based Cognitive Therapy (MBCT; Segal et al., 2002) have also been found to significantly improve sexual desire, sexual distress, and overall sexual function in an uncontrolled study (Paterson et al., 2017), and in a randomized clinical trial where improvements in sexual desire showed large effect sizes at post-treatment, and at 6- and 12-month follow-up (Cohen's $d$ ranged from 1.29 to 1.60) (Brotto et al., 2021a). Improvements in sexual distress also showed large effect sizes at all post-treatment time points (Cohen's $d$ ranged from 0.83 to 1.17). For both outcomes, the positive effects were retained 12 months later, and $58.4 \%$ of participants reported a moderate or great improvement in their overall sexual desire (Brotto et al. 2021a, b).

Despite this demonstrated efficacy, barriers to treatment access exist, including geographic location, cost, anxiety, embarrassment, and lack of available expertise by providers (Adams, 2014; Bergvall \& Himelein, 2014). As a result, only $19-32 \%$ of women with sexual dysfunction receive professional treatment (Maserejian et al., 2010; Moreira et al., 2005; Nicolosi et al., 2006). There is thus an urgent need to improve accessibility to lasting and meaningful treatment for women with sexual concerns. Web-based intervention is one way to address this need. In general, web-based therapeutic programs can reduce embarrassment, are available at low cost, are accessible to women regardless of geographic location, can be created and updated by experts (Baker et al., 2010), and can be facilitated by non-experts (Musiat \& Tarrier, 2014).

Four studies of online treatment for sexual dysfunction have reported promising effects (van Lankveld, 2016). For example, one program provided women with access to online CBT modules alongside email contact with a therapist, which led to significant improvements in sexual function that were maintained after 3 months (Jones \& McCabe, 2011). Another program combining elements of CBT with mindfulness skills found improved communication and emotional intimacy between partners, increased sexual function, and decreased distress (Hucker \& McCabe, 2014). More recently, an eight-module program for SIAD based on CBT skills has been developed. This program, called eSense$C B T$, includes engaging interactive elements plus weekly homework and has been found to be usable and enjoyed by participants (Zippan et al., 2020). The use of the program was also associated with significant improvements in sexual distress and sexual satisfaction, as well as several domains of sexual function including desire and arousal (Stephenson et al., 2021).

To extend this line of work, we have recently adapted the empirically-supported eight-session mindfulness-based treatment for SIAD (Brotto et al., 2021a) into the eSense platform. Given recent evidence of feasibility, usability, and preliminary efficacy of $e$ Sense-CBT for delivering CBT skills, the purpose of the present study was to explore the feasibility of participants completing the newly created $e$ SenseMindfulness, and to gather preliminary data on changes in clinical outcomes to power a future larger clinical trial. The 
goal of this study was to determine whether eSense-Mindfulness could be appropriate for future efficacy testing. As such, we aimed to assess feasibility domains of acceptability, implementation, practicality, and adaptation, as per the definitions established by Bowen et al. (2009). Moreover, this study included limited efficacy testing and a focus on effect sizes of change, and a shorter follow-up period (pre- to posttesting only without longer-term follow-up).

\section{Methods}

\section{Participants}

Eligible participants were drawn from a registry of women with SIAD who consented to be contacted for future research held in the lead author's research laboratory (they had previously participated in a variety of treatment-focused and laboratory-based experimental studies). In addition, we advertised on social media as well as the lead author's research website. Inclusion criteria were: self-identification as a woman (cis or trans), between the ages of 19 and 65, experiencing distress with low or absent sexual desire, having English fluency, having consistent access to the internet and basic competency in using online platforms, having a regular doctor, and being in a stable, committed romantic relationship of at least 6 months. Exclusion criteria were sexual difficulties exclusively attributable to another psychiatric diagnosis, the effect of substance use, a general medical condition, or to nonsexual conflict in a relationship. Women who self-reported a diagnosis of Provoked Vestibulodynia, or those whose sexual difficulties could be largely accounted for by their pain, were not eligible. Visual impairments that would make it difficult to read and interact with online materials were also exclusion criteria. Women receiving treatment elsewhere for their sexual concern at the time of participating in this study were also not eligible.

A total of 58 individuals expressed an interest and most of these learned about the study through a social media advertisement. Of these, $n=39$ went on to participate in a telephone screen with the research assistant. From here, $n=3$ were excluded due to experiencing significant vulvovaginal pain, $n=2$ due to not being in a relationship, and $n=1$ due to withdrawing prior to providing consent. A total of 33 women met study criteria and provided consent to participate.

The final sample included 30 participants who completed baseline measures and at least one module (three dropped out before completing any modules due to time constraints). They had an age range of 20-64 $(M=35.3, S D=9.7$; see Table 1) with 29 identifying their gender as "woman" and one identifying their gender as non-binary. All participants were partnered, and the average relationship length was 10.45 years (range $0.58-31$ years). The length of their sexual
Table.1 Participant demographic information

\begin{tabular}{ll}
\hline Characteristics & Baseline $(n=30)$ \\
\hline Age $(M, S D)$ & $35.33(9.67)$ \\
Gender identity $(N, \%)$ & $29(96.7 \%)$ \\
Woman & $1(3.3 \%)$ \\
Non-binary & \\
Sexual orientation $(N, \%)$ & $23(76.7 \%)$ \\
Heterosexual & $4(13.3 \%)$ \\
Bisexual & $1(3.3 \%)$ \\
Demisexual & $2(6.7 \%)$ \\
Pansexual & \\
Self-identified ethnicity $(N, \%)$ & $1(3.3 \%)$ \\
West Asian & $27(90.0 \%)$ \\
Caucasian & $2(6.7 \%)$ \\
Mixed & \\
Education $(N, \%)$ & $2(6.7 \%)$ \\
Highschool & $2(6.7 \%)$ \\
Attended some college & $9(30.0 \%)$ \\
Graduated college & $17(56.7 \%)$ \\
Post-graduate degree & \\
Occupational status $(N, \%)$ & $18(60.0 \%)$ \\
Employed & $3(10.0 \%)$ \\
Unemployed & $6(20.0 \%)$ \\
Student & $1(3.3 \%)$ \\
Retired & $2(6.7 \%)$ \\
Other & \\
\hline
\end{tabular}

difficulties ranged from 12 to 216 months, with an average duration of sexual difficulties of 71.6 months, or six years. Twenty-three participants identified with a heterosexual orientation, and the remaining seven as having diverse sexual attractions. This was a primarily white $(27 / 30 ; 90 \%)$ and educated (28/30; over $93 \%$ had at least some college education) sample. There was a diverse range of incomes, with a mean annual household income level at approximately $\$ 120,000$. A total of $13 / 30$ participants $(44.8 \%)$ reported a history of sexual abuse, with the majority of these experiences (37.9\% of total sample) taking place as an adult and relatively fewer taking place as a child $(6.9 \%$ of total sample). Most women in our sample (24/30; 76.7\%) had never received treatment for a sexual health difficulty.

Twenty-five women provided full pre- and post-eSense data, as well as feedback after each of the eight weekly modules. Five participants provided partial data, with two participants completing only Module 1, and two participants completing only through Module 2. Sample sizes for each quantitative outcome are provided. The groups of treatment completers and non-completers did not differ on age, relationship duration, education, duration of sexual concerns, baseline distress, and most baseline measures of sexual function, with the exception of sexual satisfaction, which was significantly higher at baseline in the treatment completers compared to the non-completers $(p=0.034)$. Among the group of 30 who consented, 26 (87\%) completed all eight modules, two completed two modules, and 
two completed only the first module. It took participants on average 11.2 weeks (SD 0.97) from the baseline questionnaire, through eSense-Mindfulness and to the post-treatment questionnaire.

\section{Procedure}

Interested participants contacted a research assistant who assessed their eligibility by phone. During this phone screen, criteria for SIAD were assessed using criteria outlined by the DSM-5 (APA, 2013) and a preliminary diagnosis was given to those who experienced at least three of six criteria plus clinically significant personal distress. Those who provided verbal consent were sent a link to an online questionnaire battery delivered by Qualtrics. The battery included demographic questions and a number of validated measures (described below).

After receiving their completed baseline questionnaires, users were sent an individualized login email to access eSense-Mindfulness. They were asked to complete one module per week for 8 weeks (on their own time), and fill out an online feedback survey about each module once it was complete. Completion of this survey triggered access to the next module. Weekly reminder emails were sent to each participant. Upon completion of the eighth module of eSenseMindfulness, participants were sent another online link to complete the post-treatment questionnaire battery as well as questions assessing their reactions to working through all eight modules of eSense-Mindfulness. Post-treatment measures were completed approximately 2 weeks after completion of the last eSense-Mindfulness module.

Participants were compensated \$25 for answering questionnaires at the pre- and post-intervention time points (\$50 total). Upon completion, participants were also given free lifetime access to eSense-Mindfulness. This study was approved by the Behavioural Research Ethics Board at the University of British Columbia and the associated hospital research ethics board at Vancouver Coastal Health Research Institute.

\section{Measures}

We assessed feasibility dimensions of acceptability (how participants reacted to eSense-Mindfulness), implementation (whether we could deliver the mindfulness treatment via an online program), practicality (the ability of a small team to deliver the intervention without providing individualized guidance to participants), adaptation (whether the mindfulness recordings could be delivered online and if participants reported engaging with them), and limited efficacy testing as per the guidelines set out by Bowen et al. (2009).
Usability Questions Per Module At the end of each of the eight modules, participants received a link with the following questions about the module they had just completed: (1) What were your overall impressions of the content you just covered? (free response); (2) To what extent do you think this particular module addressed sexual issues relevant to you? (Likert 0-10); (3) What section of the module did you find the most helpful for your sexual concerns? (check all that apply); (4) What specific content or activities from this module were the most helpful for your sexual concerns? (free response); (5) What section of the module did you find the least helpful for your sexual concerns? (check all that apply); (6) Were there any topics or content that you did not find helpful? (free response); (7) How clear was the content of this module? (Likert 0-10); (8) How easy was it for you to navigate through this module? (Likert 0-10); (9) How would you rate the look of this module? (Likert 0-10); (10) Any other comments about the look of this module? (free response); (11) How much of the audio recording(s) did you listen to? (Likert 0-10); (12) How clear was the rationale for doing the home practice(s)? (Likert 0-10); (13) How clear were the instructions for the home practice(s)? (Likert 0-10); (14) How easy or difficult was it to do the home practice(s)? (Likert 0-10); (15) How much of the assignments were you able to complete? (Likert 0-10); (16) What would have assisted you in being able to complete more or all of the home practices? (free response); and (17) Is there anything else you'd like to tell us about your experience with this module? (free response). These usability questions were previously used in similar feasibility studies of a cognitive-behavioral version of $e$ Sense (Stephenson et al., 2021; Zippan et al., 2020). Free response item responses are not included in this manuscript and were used by the development team to improve $e$ Sense for future testing.

Global Impressions of eSense-Mindfulness At the completion of Module 8, participants were sent a link to answer the following free-response questions: (1) Across all of eSense-Mindfulness, which topics or exercises did you find most relevant to addressing your sexual concerns? (2) Is there anything you learned from eSense-Mindfulness that you didn't know before? (3) Are you doing anything differently as a result of participating in this study? and (4) What is the most important thing you will take away from eSense-Mindfulness?

Sexual Function We administered the Female Sexual Function Index (FSFI; Rosen et al., 2000) as our primary measure of clinical outcomes before and after completion of eSense-Mindfulness. The FSFI includes separate subscales of sexual desire, sexual arousal, lubrication, orgasm, sexual satisfaction, and sexual pain. We modified the order of the items such that overall sexual satisfaction and sexual desire 
were assessed first, with only women reporting any solo or partnered sexual activity over the past 4 weeks continuing to answer remaining questions about sexual arousal, lubrication, orgasm, and pain with vaginal penetration. If a participant was not sexually active, they had missing scores for these domains, and thus, their other scores did not contribute to the FSFI total score. Total scores on the full-scale range from 2 to 36, with higher scores indicating better sexual function. The FSFI has been found to have good discriminant validity, correctly identifying $70.7 \%$ of women with sexual dysfunction using a cut-off score of 26.55 (Wiegel et al., 2005). In this sample, inter-item consistency using Cronbach's alpha for the FSFI total score at pre-treatment was very good at 0.87 , and inter-item consistencies for each of the domain scores ranged between 0.79 and 0.94 .

Sexual Distress We administered the Female Sexual Distress Scale-Revised (FSDS-R; Derogatis et al., 2008) before and after the completion of eSense-Mindfulness. Total scores range from 0 to 52, with higher scores indicating greater distress. The FSDS-R has been found to have excellent discriminant validity, correctly identifying $92.7 \%$ of women with hypoactive sexual desire disorder using a cut-off score of 11 (Derogatis et al., 2008). In this sample, Cronbach's alpha at baseline was 0.89 showing good inter-item consistency.

Treatment Satisfaction We administered a selection of items from the Erectile Dysfunction Inventory of Treatment Satisfaction Scale (EDITS; Althof et al., 1999; adapted for use with a female sample). Items included: (1) Overall, how satisfied are you with this treatment? (2) To what degree has this treatment met your expectations? (3) How likely are you to continue using this treatment? (4) During the past 8 weeks, how easy was it for you to use this treatment? (5) How confident has this treatment made you feel about your ability to engage in sexual activity? (6) Overall, how satisfied do you believe your partner is with the effects of this treatment? (7) How does your partner feel about your continuing to use this treatment? and (8) How natural did the process of achieving sexual response or sexual satisfaction feel when you used this treatment over the past 8 weeks? All items were asked on a 1 (e.g., very dissatisfied/not at all/very unlikely/very difficult) to 5 (e.g., very satisfied/completely/ very likely/very easy) Likert scale with higher scores representing more satisfaction.

Homework Completion We administered a selection of items from the Homework Rating Scale focused on compliance with the prescribed homework exercises (Kazantzis et al., 2004). These included the following: (1) How much of the assignments were you able to do? (2) How well did you do the assignments? (3) How difficult were the assignments? (4) How much did obstacles interfere with the assignments?
(5) How well did you understand what to do? (6) How well did you understand the reason for doing the assignments? (7) How much involvement did you have in planning the assignment? (8) How specific were the guidelines on how to do the assignments? (9) How well did the assignments match your therapy goals? (10) How much did you enjoy the assignments? (11) How much did the assignments help you gain control over your problems? and (12) Did the assignments help with your progress in treatment? All items were assessed on a 0 (e.g., none/not at all) to 4 (e.g., extensive/extremely) Likert scale, with higher scores indicating more engagement and positive experiences with homework assignments.

\section{eSense-Mindfulness Content}

The content of eSense-Mindfulness was adapted from an efficacious face-to-face mindfulness intervention for women with SIAD (Brotto et al., 2021a; Paterson et al., 2017). It housed eight modules which progressed through different mindfulness exercises that participants could practice regularly on their own by following downloadable audio guides. No individualized guidance by a team member was provided to participants. It incorporated three rich case examples to show the progression of fictional (but realistic) characters with SIAD as they learned and applied mindfulness skills. eSense-Mindfulness also delivered basic psychoeducation about the prevalence of sexual concerns and models of sexual response.

In line with best practices for online therapies, text was interspersed with pictures, diagrams, videos, and audio clips to maximize engagement. Each module introduced a new structured mindfulness practice delivered by an embedded audio file. Formal practices over the eight modules included: eating meditation, breathing space, body scan, walking meditation, mindfulness of thoughts, working with difficulty meditation, mindfulness of sensations, sexual sensations meditation, and pleasurable touch meditation. A stretch and breath mindfulness was demonstrated by video file. Three fictional cases were introduced in Module 1 and were used to illustrate how mindfulness practices could be implemented, as well as address challenges that arise. In addition, each case was used to illustrate the formal Inquiry process by showing characters' reactions to three key questions: (1) What did you notice in this practice? (2) How was paying attention in this practice different from how you normally pay attention? and (3) How was this mindfulness practice relevant to your sexual concerns? Each module also delivered a small amount of information about sexuality including, for example, the prevalence of sexual difficulties, models of sexual response, causes of sexual difficulties, body image, sexual beliefs, communication skills, an overview of other treatments, and a potential plan for ongoing practice. 
See Table 2 for a summary of eSense-Mindfulness content. Participants only interacted with a member of the study team to complete a telephone screening and diagnostic interview prior to beginning. Participants completed the eight modules of eSense-Mindfulness over the course of 8 to 12 weeks.

\section{Data Analyses}

We focused on the mean responses to each of the usability questions (rated 0-10) for each module (Table 3). To examine whether there were differences in the self-reports of usability across modules, we also carried out a repeated measures within-subjects analysis of variance (ANOVA). Paired samples t-tests were used to compare pre to post scores on domains of the FSFI and the FSDS-R. The degree to which participants completed the recommended homework practices provided an index of practicality, or the extent to which eSense could be delivered with limited resources, and without individualized guidance. For self-reported homework completion using the Homework Rating Scale, we report on the proportion of participants who were able to complete "a lot" or "all" of the recommended practices. All data are available at the Open Science Framework https:/osf.io/ r3my9/

\section{Results}

\section{Baseline Clinical Scores}

At baseline, all participants $(n=30)$ scored within the clinical range of sexual distress on the FSDS-R (Derogatis et al., 2008), and 23 participants (96.2\%) scored in the clinical range on overall sexual function using the FSFI total score (Wiegel et al., 2005).

\section{Acceptability}

In response to the question asking about whether the participant felt that the module addressed sexual issues that were relevant, every score was greater than 7.0 (out of 10) with a significant increase in relevance as the modules progressed, $F(7,147)=2.75, p=0.01$. Responses to the question asking about how clear the module was were all greater than 8.7 (out of 10) with no significant difference in scores across the modules $(p>0.05)$. We then asked about how easy it was to navigate through the modules, and there was no difference in ratings across the modules $(p>0.05)$, with all scores greater than 8.3 (out of 10). In general, participants reported liking the look of the modules, with all scores greater than 7.7

Table.2 Structure of eSense-Mindfulness content across eight modules

\begin{tabular}{|c|c|}
\hline Module & Content \\
\hline 1: Definitions and Causes of Sexual Dysfunction & $\begin{array}{l}\text { Introduction to sexual function } \\
\text { Causes of sexual dysfunction } \\
\text { Theoretical models of sexual dysfunction } \\
\text { Introduction to mindfulness: Mindful Eating }\end{array}$ \\
\hline 2: Increasing Awareness of Physical Sensations & $\begin{array}{l}\text { A rationale for mindfulness in sexual difficulties } \\
\text { How mindfulness fits within the cognitive model } \\
\text { Body Scan practice }\end{array}$ \\
\hline 3: Exploring the Body and Judgments About it & $\begin{array}{l}\text { Mindful Stretching } \\
\text { Walking Meditation } \\
\text { Addressing body image }\end{array}$ \\
\hline 4: Awareness of Sexual Thoughts and Beliefs & $\begin{array}{l}\text { The role of thoughts and beliefs in sex } \\
\text { A mindful approach to thoughts } \\
\text { Mindfulness of thoughts meditation }\end{array}$ \\
\hline 5: Working with Aversion and Self-Touch & $\begin{array}{l}\text { Aversion and avoidance } \\
\text { Working with difficulty meditation } \\
\text { Introduction to self-touch }\end{array}$ \\
\hline 6: Creating Awareness of Sexual Sensations & $\begin{array}{l}\text { Fantasy, erotica, and vibrators as sexual stimuli } \\
\text { Sexual sensations meditation } \\
\text { Pleasurable touch exercise } \\
\text { Sexual communication }\end{array}$ \\
\hline 7: Sensate Focus with Your Partner & $\begin{array}{l}\text { Introduction to sensate focus with a partner } \\
\text { Positive communication skills } \\
\text { Structure of couple sensate focus }\end{array}$ \\
\hline 8: Maintaining (and Extending) Your Gains & $\begin{array}{l}\text { Summary of program components and rationale } \\
\text { Focus on contextual maintaining factors and skills checklist } \\
\text { Relapse prevention } \\
\text { Additional treatment options }\end{array}$ \\
\hline
\end{tabular}




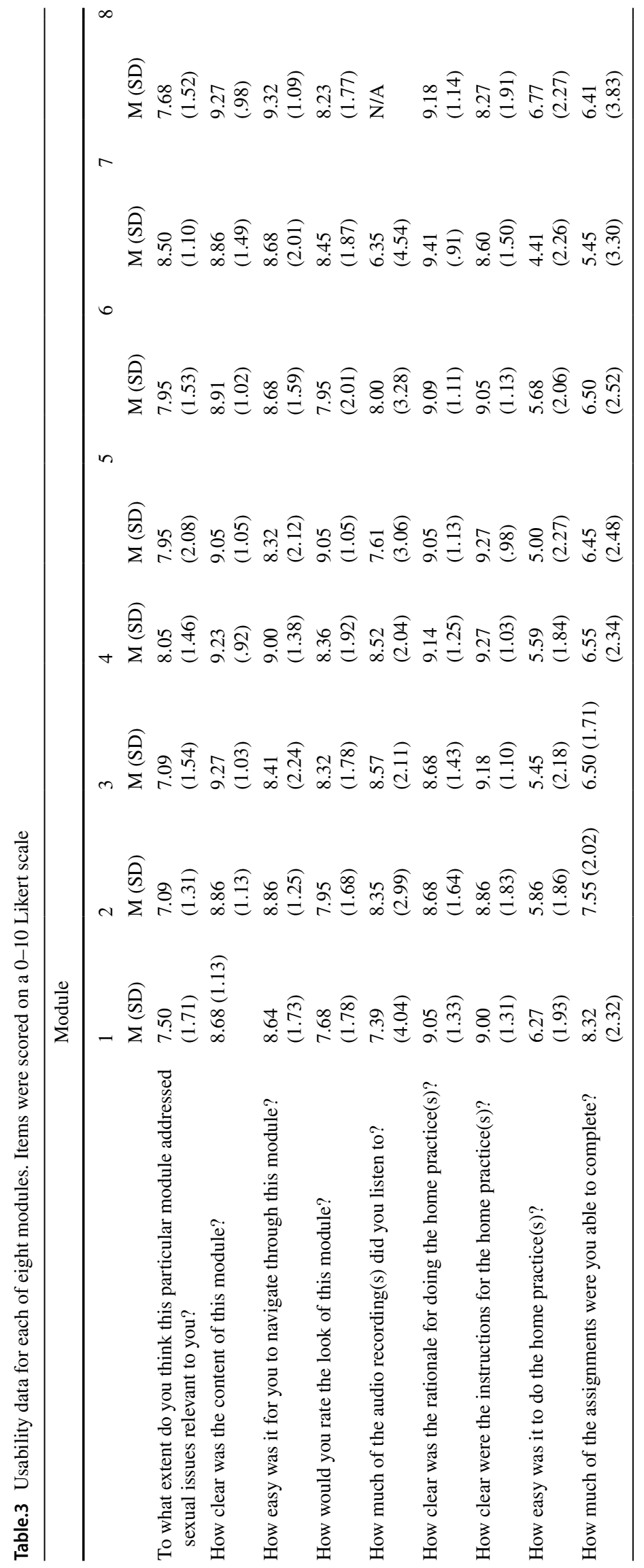




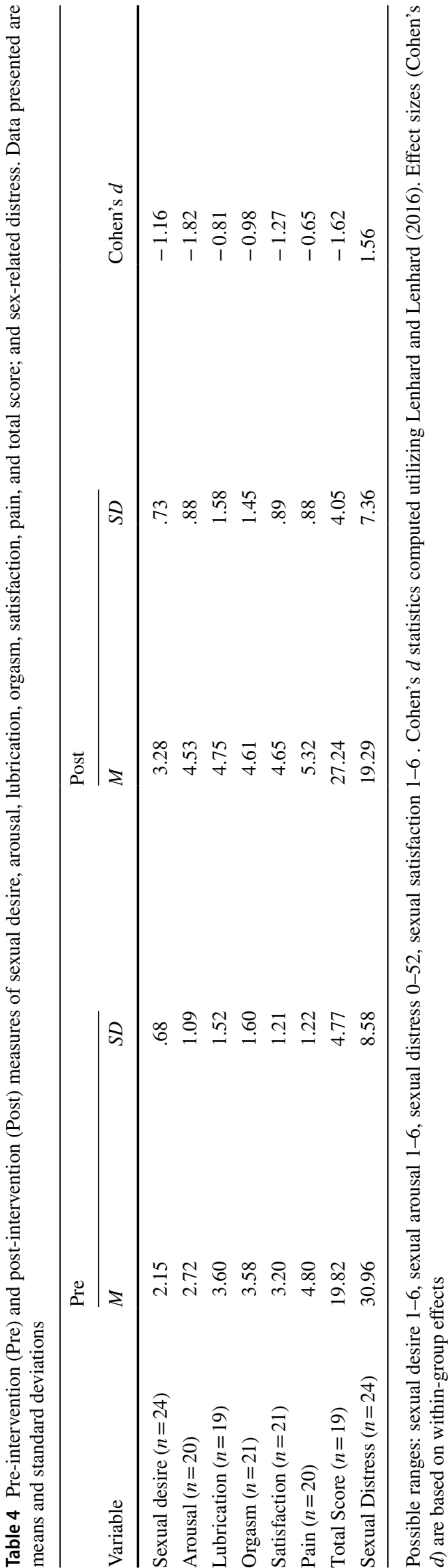

(out of 10) and an increase in scores with later modules, $F(7,147)=3.68, p=0.001$. The lowest score for the question asking how much of the audio recordings were listened to was 6.34 (out of 10), with no change in ratings across modules $(p>0.05)$. Participants found that the rationale provided for the various homework exercises was compelling, with all weekly scores being 8.7 or greater (out of 10), and no significant change in ratings of clarity over the modules $(p>0.05)$. They also found the homework instructions to be very clear, with scores at least 8.3 (out of 10), and a decrease in scores with later modules $F(7,147)=2.27, p=0.032$. Ratings for how easy participants found the homework were overall lower, with the lowest module (7) being rated as 4.41 (out of 10), and an overall decrease across modules in how easy participants found the homework to be, $F(7,147)=3.45$, $p=0.002$. Most of the homework was completed for module 1 (8.32 out of 10), but then decreased over the course of eSense-Mindfulness, $F(7,147)=3.56, p=0.001$.

\section{Limited Efficacy Testing}

A total of 24 participants had complete FSFI data at baseline, and 20 had FSFI data at post-test (Table 4). Scores on all domains of the FSFI significantly increased over time: sexual desire, $t(23)=-5.66, p<0.001$, Cohen's $d=-1.16$; sexual arousal, $t(19)=-8.12, p<0.001$, Cohen's $d=-1.82$; lubrication, $t(18)=-3.55, p=0.002$, Cohen's $d=-0.81$; orgasm, $t(20)=-4.50, p<0.001$, Cohen's $d=-0.98$; sexual satisfaction, $t(20)=-5.81, p<0.001$, Cohen's $d=-1.27$; sexual pain, $t(19)=-2.90, p=0.009$, Cohen's $d=-0.65$; and sexual function total score, $t(18)=-7.05, p<0.001$, Cohen's $d=-1.62$. Thirty percent of the sample remained below the clinical cut-off on the FSFI total score at posttreatment. A paired samples t-test on sexual distress scores from pre- to post was statistically significant, $t(23)=7.62$, $p<0.001$, Cohen's $d=1.56$. A total of $91.7 \%$ of participants remained in the clinical range on this measure of sexual distress.

\section{Adaptation}

We used the EDITS to capture participant satisfaction across eight questions, which provided an indication of success in adapting the face-to-face mindfulness protocol to online delivery. As shown in Table 5, participants reported a very high level of satisfaction with the following: the overall treatment; likelihood of using this treatment; confidence about engaging in sexual activity; and partner's feelings about participant using the treatment. They reported that eSense-Mindfulness "considerably" met expectations and that partners were "somewhat satisfied" with the effects of treatment. They reported that it was "neither easy nor difficult" to use eSense-Mindfulness. 
Table.5 Responses to treatment satisfaction questions. Data presented are means and standard deviations

\begin{tabular}{lc}
\hline Item & Mean (SD) \\
\hline Overall, how satisfied are you with this treatment & $4.38(0.77)$ \\
To what degree has this treatment met your expectations? & $3.92(0.50)$ \\
How likely are you to continue using this treatment? & $4.42(0.83)$ \\
During the past 8 weeks, how easy was it for you to use this treatment? & $2.96(1.08)$ \\
How confident has this treatment made you feel about your ability to engage in sexual activ- & $4.13(0.45)$ \\
ity? & $3.91(0.85)$ \\
Overall, how satisfied do you believe your partner is with the effects of this treatment? & $4.17(0.78)$ \\
How does your partner feel about your continuing to use this treatment? & $3.83(0.82)$ \\
How natural did the process of achieving sexual response or sexual satisfaction feel when you & \\
\hline
\end{tabular}

Items were rated on a 1 to 5 Likert scale with higher scores reflecting more satisfaction

\section{Practicality}

Fifty percent of the sample reported being able to do a lot or all of the homework; $30 \%$ reported being able to do the assignments well; $58.3 \%$ reported finding the home practices at least moderately difficult; and $70.8 \%$ said that obstacles at least moderately interfered with the practices. All participants reported a full understanding of the assignments, as well as understanding the underlying reasons. Over $95 \%$ felt that the assigned practices matched their therapy goals and $87.5 \%$ reported enjoying the practices. A total of $83.3 \%$ felt that the assignments helped them gain control over their problems and $91 \%$ felt that the assigned practices helped with their progress in treatment.

\section{Impacts of COVID-19}

Although the study took place during the global COVID-19 pandemic, $68.2 \%$ of the participants reported "no impact" of the pandemic on their participation in this study. Among those who reported that the pandemic impacted their participation, the most common reason was having to take care of children.

\section{Discussion}

The current study assessed the feasibility of eSense-Mindfulness, a new online treatment for women with sexual interest/ arousal disorder. Thirty women were invited to complete an eight-module online mindfulness-based intervention, without individualized guidance, during the COVID-19 pandemic. We evaluated the intervention for several domains of feasibility, including acceptability, implementation, practicality, adaptation, as well as limited efficacy testing. All assessed domains suggested that the program was highly feasible and that our adaptation from a face-to-face mindfulness program to online delivery was practical. Participants were generally satisfied with the platform's appearance and were able to use eSense-Mindfulness effectively. Users reported a high level of satisfaction with clarity and relevance of the content, along with clarity of the homework instructions and rationale, which increased as they progressed through the modules. However, ratings for ease of completing the home exercises were lower. Despite this difficulty, attrition was fairly low ( 25 of the 30 women who provided baseline data completed all assessments and modules, with the remaining five providing partial data), and in line with typical rates for smartphone-based apps delivering unguided mental health interventions where attrition is often 20-25\% (Linardon \& Fuller-Tyszkiewicz, 2020).

Acceptability was assessed in terms of participant satisfaction, and they reported that eSense-Mindfulness "considerably" met expectations and that their partners were "somewhat satisfied" with the effects of treatment. Although the women needed to be in committed relationships to participate, the program was designed to be an individual, not couples-based intervention, which may explain partners' somewhat lower rating. Indeed, couples-focused mindfulness sex programs show that including one's partner and including mindfulness exercises that focus on the couple can be effective for relationship satisfaction and communication (Leavitt et al., 2021). Results from limited efficacy testing were promising. Participants' sexual function (desire, sexual arousal, lubrication, orgasm, and pain) and satisfaction improved significantly while distress decreased significantly, all with large effect sizes. Although a control group would be needed to make any firm conclusions about efficacy, women with low desire rarely exhibit significant improvement in waitlist conditions (e.g., Brotto \& Basson, 2014), making it fairly unlikely that the improvements seen here can be attributed to the passage of time alone. Thus, the mindfulness exercises in eSense-Mindfulness were found to be highly feasible, usable, and potentially effective.

Given that we translated topics from mindfulnessbased group therapy that are usually explained by a group 
facilitator, one domain of feasibility we were particularly interested in was adaptation or the extent to which an established intervention can be adapted for online use. It is noteworthy that these concepts could be described in an accessible and engaging way through images, audio, and text only. Usability ratings were high (all scores greater than 7 out of 10), with the exception of those around the difficulty of homework completion. The average score rating how relevant women found the material was high, increasing as users progressed through the modules, and scores rating the clarity were consistently very high. The navigation and look of the modules were also rated very positively.

However, although users found the home practice instructions to be very clear and rated the provided rationales highly, ratings of homework completion were lower. Only fifty percent reported being able to do most or all of the homework, and over $70 \%$ also reported obstacles to doing homework. These results may speak to the volume of homework being too much-participants had difficulty finding enough time in their busy schedules to accommodate the recommended amount of practice. This challenge with homework compliance is also found in briefer (i.e., two sessions) mindfulness programs addressing sexuality delivered in a couples format (Leavitt et al., 2021). In addition to a lack of time as the primary obstacle, participants also noted needing more privacy, childcare, accountability, and automated reminders. The finding that it took on average 11.2 weeks to complete both assessment questionnaires and the eSense-Mindfulness program might relate to the volume of home activities recommended.

Experts have made various recommendations for how to improve adherence to recommended mindfulness practices during e-mindfulness programs. For example, supplementing online mindfulness with therapist support, either from a live therapist or automated responses, may improve homework adherence (Spijkerman et al., 2016). Others have shown that online mindfulness programs that recommend more frequent but shorter mindfulness practices each day, as opposed to a single longer one, may improve adherence, and also encouraging users to pay attention to the quality of their mindfulness practice rather than the quantity of it, may also improve engagement with homework (Berghoff et al., 2017). Interestingly, when a coach or navigator is available to check in with participants throughout their program, research suggests that even individuals with no therapy training who are only trained on helping participants navigate the platform can significantly improve engagement and outcomes (Baumeister et al., 2014; Robinson et al., 2010; Titov et al., 2010). These and other changes will be considered in future iterations of eSense-Mindfulness.

While we undertook limited efficacy testing as one indicator of the feasibility of eSense-Mindfulness, the study was intentionally not powered to detect efficacy, nor to identify which components of the program participants found most useful. Nonetheless, several participants provided unsolicited feedback that they found the mindfulness skills and psychoeducational material to be the most helpful components of eSense-Mindfulness. Some also reported that learning about communication skills was very helpful; this content was adapted from widely used methods of couple therapy (e.g., Christensen et al., 2004; Gottman, 1999), and the infusion of mindfulness into these communication skills exercises potentially meant that participants may have communicated more intentionally and mindfully than is typical.

Limited efficacy testing in this study revealed large effect sizes (i.e., Cohen's $d>0.80$ ) for every domain of sexual function (except pain) and sex-related distress. These effect sizes were similar to those found when a group of women with SIAD participated in a comparable online CBT program (Stephenson et al., 2021; Zippan et al., 2020). Effect sizes were also in line with in-person mindfulness-based therapies for sexual dysfunction (e.g., Brotto et al., 2021a; Paterson et al., 2017).

There is a considerable body of research supporting the feasibility (e.g., Krusche et al., 2013; Lengacher et al., 2018; Morledge et al., 2013), usability (e.g., Cillessen et al., 2020; Stjernswärd \& Hansson, 2017), and efficacy (Jayawardene et al., 2017; Spijkerman et al., 2016) of online mindfulness programs for a variety of conditions including anxiety, stress, coping with cancer, depression, multiple sclerosis, and a host of other issues. Collectively, these studies (and the much larger literature in online mindfulness interventions) find that adherence predicts outcomes, that such programs are highly accessible, and that effect sizes for primary clinical outcomes tend to be in the moderate range. Given the demonstrated efficacy of in-person mindfulness interventions for women's sexual concerns (Stephenson, 2017), and the present preliminary data on feasibility and efficacy when such programs are adapted for online delivery, we would predict that a larger evaluation of the efficacy of eSense-Mindfulness would demonstrate its positive effects on clinical outcomes relevant to women with SIAD.

The ongoing COVID-19 pandemic has exacerbated existing barriers and worsened sexual problems ( $\mathrm{Li}$ et al., 2020; Wind et al., 2020), highlighting the need for more online therapy options. It has also increased gender inequality in terms of both income and mental health problems (including those that contribute to sexual dysfunction), with women being more likely than men to experience such disparities (Brotto et al., 2021b). Thus, programs like eSense-Mindfulness can serve as adjuncts to more traditional forms of therapy (acting as an additional resource for those already in treatment), as a 
stopgap in therapy that has been delayed (e.g., VillemaireKrajden \& Myhr, 2019), or as a method of increasing willingness to access to therapy by reducing stigma (e.g., Levin et al., 2018).

\section{Limitations and Future Research}

There were a number of limitations, however, that need to be kept in mind. First, the study did not include a control condition, which meant that we could not confidently attribute improvements over time to use of eSense-Mindfulness. Future efficacy testing will require a comparison group to rule out the general passage of time or other nonspecific factors as alternative causes of improvement. Second, we employed a relatively small convenience sample, rendering it unknown whether results would generalize to the broader population of women with SIAD. For example, those who elected to participate may have been more motivated than the larger population of women with SIAD, as treatment seekers tend to have a longer duration of low desire (Maserejian et al., 2010). Though the present findings provided evidence of successful implementation, whether eSense-Mindfulness could be implemented with a more diverse sample requires further testing. In addition, this sample consisted primarily of highly educated, moderately affluent Caucasian women. Populations with female sexual dysfunction are likely more diverse in terms of education level, income, and ethnicity (e.g., Ojeda \& McGuire, 2006; Walker et al., 2015); future studies should ideally recruit samples with reduced access to individualized sex therapy. Our sample was also relatively technologically fluent and may not be representative of the population that might access a future, publicly available version of $e$ Sense-additional technical supports may be needed for some users. We were also unable to directly assess participant engagement with $e$ Sense-Mindfulness using website analytics; therefore, the degree of objective user engagement with the various components of modules is unknown. Future studies that directly assess engagement may identify components that are used at higher or lower rates, which can help to reshape eSense-Mindfulness content.

Author Contribution LAB: designed and executed the study, assisted with the data analyses, wrote the paper, and participated in final editing. KRS: collaborated with the design, analysis, writing, and editing of the manuscript. NZ: lead the study methods, collaborated on the writing of the manuscript, and proofread at all stages.

\section{Declarations}

Ethics Statement This study was approved by the Behavioural Research Ethics Board at the University of British Columbia and the associated hospital research ethics board at Vancouver Coastal Health Research Institute.

Informed Consent Each of the participants provided verbal and electronic signed consent to participate.

Conflict of Interest The authors declare no competing interests.

\section{References}

Adams, S. A. (2014). Marital quality and older men's and women's comfort discussing sexual issues with a doctor. Journal of Sex \& Marital Therapy, 40, 123-138.

Althof, S., Corty, E., Levine, S., Levine, F., Burnett, A. L., McVary, K., Stecher, V., \& Seftel, A. D. (1999). EDITS: The development of questionnaires for evaluating satisfaction with treatments for erectile dysfunction. Urology, 53(4), 793-799.

American Psychiatric Association. (2013). Diagnostic and statistical manual of mental disorders (5th ed.). Washington, DC.

Baker, T. B., Gustafson, D. H., Shaw, B., Hawkins, R., Pingree, S., Roberts, L., \& Strecher, V. (2010). Relevance of CONSORT reporting criteria for research on eHealth interventions. Patient Education and Counseling, 81, S77-S86.

Barlow, D. H. (1986). Causes of sexual dysfunction: The role of anxiety and cognitive interference. Journal of Consulting and Clinical Psychology, 54, 140-148.

Baumeister, H., Reichler, L., Munzinger, M., \& Lin, J. (2014). The impact of guidance on internet-based mental health interventions-A systematic review. Internet Interventions, 1, 205-215.

Berghoff, C. R., Wheeless, L. E., Ritzert, T. R., et al. (2017). Mindfulness meditation adherence in a college sample: Comparison of a 10-Min Versus 20-Min 2-Week Daily Practice. Mindfulness, 8, $1513-1521$.

Bergvall, L., \& Himelein, M. J. (2014). Attitudes toward seeking help for sexual dysfunctions among US and Swedish college students. Sexual and Relationship Therapy, 29(2), 215-228.

Bowen, D. J., Kreuter, M., Spring, B., Cofta-Woerpel, L., Linnan, L., Weiner, D., Bakken, S., Kaplan, C., Squiers, L., \& Fernandez, M. (2009). How we design feasibility studies. American Journal of Preventive Medicine, 36, 452-457.

Brotto, L. A., \& Basson, R. (2014). Group mindfulness-based therapy significantly improves sexual desire in women. Behaviour Research and Therapy, 57, 43-54.

Brotto, L. A., \& Heiman, J. R. (2007). Mindfulness in sex therapy: Applications for women with sexual difficulties following gynecologic cancer. Sexual and Relationship Therapy, 22, 3-11.

Brotto, L. A., Basson, R., \& Luria, M. (2008a). A mindfulness-based group psychoeducational intervention targeting sexual arousal disorder in women. Journal of Sexual Medicine, 5, 1646-1659.

Brotto, L. A., Heiman, J. R., Goff, B., Greer, B., Lentz, G., Swisher, E., Tamimi, H., \& Van Blaricom, A. (2008b). A psychoeducational intervention for sexual dysfunction in women with gynecological cancer. Archives of Sexual Behavior, 37, 317-329.

Brotto, L.A., Zdaniuk, B., Chivers, M.L., Jabs, F., Grabovac, A., Lalumiere, M.L., Weinberg, J., Schonert-Reichl, K., \& Basson, R. (2021). A randomized trial comparing group mindfulness-based cognitive therapy with group supportive sex education and therapy for the treatment of female sexual interest/arousal disorder. Manuscript under review.

Brotto, L. A., Chankasingh, K., Baaske, A., Albert, A., Booth, A., Kaida, A., Smith, L. W., Racey, S., Gottschlich, A., Murray, M. C. M., Sadarangani, M., Ogilvie, G. S., \& Galea, L. (2021). The influence of sex, gender, age, and ethnicity on psychosocial factors 
and substance use throughout phases of the COVID-19 pandemic. PLoS One, 16(11), e0259676.

Chivers, M. L., \& Brotto, L. A. (2017). Controversies of women's sexual arousal and desire. European Psychologist, 22, 5-26.

Christensen, A., Atkins, D. C., Berns, S., Wheeler, J., Baucom, D. H., \& Simpson, L. E. (2004). Traditional versus integrative behavioral couple therapy for significantly and chronically distressed married couples. Journal of Consulting and Clinical Psychology, 72(2), 176-191.

Cillessen, L., van de Ven, M. O., Compen, F. R., Bisseling, E. M., van der Lee, M. L., \& Speckens, A. E. (2020). Predictors and effects of usage of an online mindfulness intervention for distressed cancer patients: Usability study. Journal of Medical Internet Research, 22 , e17526.

DeRogatis, L., Clayton, A., Lewis-D'Agostino, D., Wunderlich, G., \& Fu, Y. (2008). Validation of the Female Sexual Distress ScaleRevised for assessing distress in women with Hypoactive Sexual Desire Disorder. Journal of Sexual Medicine, 5, 357-364.

Dewitte, M. (2016). Gender differences in implicit processing of sexual stimuli. European Journal of Personality, 30, 107-124.

Gottman, J. M. (1999). The marriage clinic: A scientifically based marital therapy. W. W. Norton \& Co.

Hucker, A., \& McCabe, M. P. (2014). An online, mindfulness-based, cognitive-behavioural therapy for female sexual difficulties: Impact on relationship functioning. Journal of Sex \& Marital Therapy, 40, 561-576.

Jayawardene, W. P., Lohrmann, D. K., Erbe, R. G., \& Torabi, M. R. (2017). Effects of preventive online mindfulness interventions on stress and mindfulness: A meta-analysis of randomized controlled trials. Preventive Medicine Reports, 5, 150-159.

Jones, L. M., \& McCabe, M. P. (2011). The effectiveness of an Internet-based psychological treatment program for female sexual dysfunction. Journal of Sexual Medicine, 8, 2781-2792.

Kazantzis, N., Deane, F. P., \& Ronan, K. R. (2004). Assessing compliance with homework assignments: Review and recommendations for clinical practice. Journal of Clinical Psychology, 60, 627-641.

Krusche, A., Cyhlarova, E., \& Williams, J. M. G. (2013). Mindfulness online: an evaluation of the feasibility of a web-based mindfulness course for stress, anxiety and depression. $B M J$ Open, 3, e003498.

Leavitt, C. E., Whiting, J. B., \& Hawkins, A. J. (2021). The sexual mindfulness project: An initial Presentation of the sexual and relational associations of sexual mindfulness. Journal of Couple and Relationship Therapy, 20, 32-49.

Lengacher, C. A., Reich, R. R., Ramesar, S., Alinat, C. B., Moscoso, M., Cousin, L., Marino, V. R., Elias, M. N., Paterson, C. L., Pleasant, M. L., Rodriguez, C. S., Wang, H., Kip, K. E., Meng, H., \& Park, J. Y. (2018). Feasibility of the mobile mindfulness-based stress reduction for breast cancer (mMBSR (BC)) program for symptom improvement among breast cancer survivors. Psycho-Oncology, 27, 524-531.

Levin, M. E., Krafft, J., \& Levin, C. (2018). Does self-help increase rates of help seeking for student mental health problems by minimizing stigma as a barrier? Journal of American College Health, 66(4), 302-309. https://doi.org/10.1080/07448481.20 18.1440580

Li, G., Tang, D., Song, B., Wang, C., Qunshan, S., Xu, C., Geng, H., Wu, H., He, X., \& Cao, Y. (2020). Impact of the COVID-19 pandemic on partner relationships and sexual and reproductive health: Cross-sectional, online survey study. Journal of Medical Internet Research, 22, e20961.

Linardon, J., \& Fuller-Tyszkiewicz, M. (2020). Attrition and adherence in smartphone-delivered interventions for mental health problems: A systematic and meta-analytic review. Journal of Consulting and Clinical Psychology, 88, 1-13.
Maserejian, N. N., Parish, S., Shifren, J. L., Huang, L., Gerstenberger, E., \& Rosen, R. C. (2010). Healthcare utilization in women diagnosed with hypoactive sexual desire disorder: Interim baseline results from the HSDD Registry for Women. Journal of Women's Health, 19, 2001-2009.

Mitchell, K. R., Jones, K. G., Wellings, K., Johnson, A. M., Graham, C. A., Datta, J., \& Macdowall, W. (2016). Estimating the prevalence of sexual function problems: The impact of morbidity criteria. Journal of Sex Research, 53, 955-967.

Mitchell, K. R., Mercer, C. H., Ploubidis, G. B., Jones, K. G., Datta, J., Field, N., Copas, A. J., Tanton, C., Erens, B., Sonnenberg, P., Clifton, S., Macdowall, W., Phelps, A., Johnson, A. M., \& Wellings, K. (2013). Sexual function in Britain: Findings from the third National Survey of Sexual Attitudes and Lifestyles (Natsal-3). Lancet, 382, 1817-1829.

Moreira, E. D., Brock, G., Glasser, D. B., Nicolosi, A., Laumann, E. O., Paik, A., Wang, T., \& Gingell, C. (2005). Help-seeking behaviour for sexual problems: The global study of sexual attitudes and behaviors. International Journal of Clinical Practice, $59,6-16$.

Morledge, T. J., Allexandre, D., Fox, E., Fu, A. Z., Higashi, M. K., Kruzikas, D. T., Pham, S. V., \& Reese, P. R. (2013). Feasibility of an online mindfulness program for stress management-A randomized, controlled trial. Annals of Behavioral Medicine, 46, 137-148.

Musiat, P., \& Tarrier, N. (2014). Collateral outcomes in e-mental health: A systematic review of the evidence for added benefits of computerized cognitive behavior therapy interventions for mental health. Psychological Medicine, 44, 3137-3150.

Nicolosi, A., Laumann, E. O., Glasser, D. B., Brock, G., King, R., \& Gingell, C. (2006). Sexual activity, sexual disorders and associated help-seeking behavior among mature adults in five anglophone countries from the Global Study of Sexual Attitudes and Behaviors (GSSAB). Journal of Sex \& Marital Therapy, 32, 331-342.

Nobre, P., \& Pinto-Gouveia, J. (2006). Dysfunctional sexual beliefs as vulnerability factors for sexual dysfunction. Journal of Sex Research, 43, 68-75.

Ojeda, V. D., \& McGuire, T. G. (2006). Gender and racial/ethnic differences in use of outpatient mental health and substance use services by depressed adults. Psychiatric Quarterly, 77, 211-222.

Paterson, L. Q. P., Handy, A. B., \& Brotto, L. A. (2017). A pilot study of 8-session mindfulness-based cognitive therapy adapted for women's sexual interest/arousal disorder. Journal of Sex Research, 54, 850-861.

Robinson, E., Titov, N., Andrews, G., McIntyre, K., Schwencke, G., \& Solley, K. (2010). Internet treatment for generalized anxiety disorder: A randomized controlled trial comparing clinician vs technician assistance. PloS One, 5, e10942.

Rosen, R., Brown, C., Heiman, J., Leiblum, S., Meston, C., Shabsigh, R., Ferguson, D., \& D'Agostino, R. (2000). The Female Sexual Function Index (FSFI): A multidimensional self-report instrument for the assessment of female sexual function. Journal of Sex \& Marital Therapy, 26, 191-208.

Segal, Z. V., Williams, J. M. G., \& Teasdale, J.D. (2002). Mindfulness-based cognitive therapy for depression: A new approach to preventing relapse. Guilford Press.

Spijkerman, M. P. J., Pots, W. T. M., \& Bohlmeijer, E. T. (2016). Effectiveness of online mindfulness-based interventions in improving mental health: A review and meta-analysis of randomised controlled trials. Clinical Psychology Review, 45, 102-114.

Stephenson, K. R. (2017). Mindfulness-based therapies for sexual dysfunction: A review of potential theory-based mechanisms of change. Mindfulness, 8, 527-543. 
Stephenson, K. R., \& Kerth, J. (2017). Effects of mindfulness-based therapies for female sexual dysfunction: A meta-analytic review. Journal of Sex Research, 54, 832-849.

Stephenson, K. R., Zippan, N., \& Brotto, L. A. (2021). Feasibility of a cognitive-behavioral online intervention for women with sexual interest/arousal disorder. Journal of Clinical Psychology, 77, 1877-1893.

Stjernswärd, S., \& Hansson, L. (2017). Effectiveness and usability of a web-based mindfulness intervention for families living with mental illness. Mindfulness, 8, 751-764.

Titov, N., Andrews, G., Davies, M., McIntyre, K., Robinson, E., \& Solley, K. (2010). Internet treatment for depression: A randomized controlled trial comparing clinician vs. technician assistance. PloS One, 5, e10939.

van Lankveld, J. (2016). Internet-based interventions for women's sexual dysfunction. Current Sexual Health Reports, 8, 136-143.

Villemaire-Krajden, R., \& Myhr, G. (2019). Evaluating the use of a computerized CBT program for outpatients on a waitlist in a university CBT unit. Journal of Psychiatric Practice, 25, 268-278.

Walker, E. R., Cummings, J. R., Hockenberry, J. M., \& Druss, B. G. (2015). Insurance status, use of mental health services, and unmet need for mental health care in the United States. Psychiatric Services, 66, 578-584.

Wiegel, M., Meston, C., \& Rosen, R. (2005). The female sexual function index (FSFI): Cross-validation and development of clinical cufoff scores. Journal of Sex \& Marital Therapy, 31, 1-20.

Wind, T. R., Rijkeboer, M., Andersson, G., \& Riper, H. (2020). The COVID-19 pandemic: The 'black swan' for mental health care and a turning point for e-health. Internet interventions, $20,100317$.

Zippan, N., Stephenson, K. R., \& Brotto, L. A. (2020). Feasibility of a brief online psychoeducational intervention for women with sexual interest/arousal disorder. Journal of Sexual Medicine, $17,2208-2219$.

Publisher's Note Springer Nature remains neutral with regard to jurisdictional claims in published maps and institutional affiliations. 